\title{
Microsurgical approach for unusual and unexpected malignant fibrous histiocytoma of the forearm: A case report
}

\author{
VALERIO D'ORAZI ${ }^{1}$, ANDREA PANUNZI $^{1}$, MARCO PAOLONI $^{2}$, ALICE ORTENSI $^{1}$, SILVIA ANICHINI $^{1}$, \\ MICHELA CIALINI $^{1}$, STEFANO NARDONI ${ }^{3}$, PAOLO URCIUOLI ${ }^{4}$, VITTORIO PASTA ${ }^{4}$ and ANDREA ORTENSI ${ }^{1}$ \\ 1 Department of General Microsurgery and Hand Surgery, Fabia Mater Hospital, I-00171 Rome;
${ }^{2}$ Biomechanics and Movement Analysis Laboratory, Physical Medicine and Rehabilitation, Sapienza University, I-00161 Rome;
${ }^{3}$ Pathology Service S.A.MAR., I-00167 Rome; ${ }^{4}$ Department of Surgical Sciences, Sapienza University, I-00161 Rome, Italy
}

Received June 12, 2015; Accepted July 17, 2015

DOI: $10.3892 / \mathrm{mco} .2015 .618$

\begin{abstract}
Soft tissue sarcomas are rare tumors with a dismal prognosis. Among the most common histological types of sarcomas of the extremities, malignant fibrous histiocytoma (MFH) is the one with the highest incidence. Surgery is considered to be the first choice of treatment for MFH. To the best of our knowledge, this is the first case report in the literature of a patient with MFH within the abductor pollicis longus (APL) muscle. This unusual location was also unexpected by the treating surgeons, as the preoperative magnetic resonance imaging localized the tumor inside a different muscle. A 79-year-old Caucasian man presented with a swelling in the middle third of the dorsal aspect of the left forearm. MFH was diagnosed following biopsy and instrumental diagnostic examinations. Surgical excision and simultaneous reconstruction was performed by the same microsurgical team, achieving an excellent functional outcome. The present case highlights the significance of microsurgical approach for improving strategic planning in oncologic surgery. Accurate surgical dissection, performed by a team of microsurgeons, allowed for the identification of the unusual and unexpected tumor localization within the APL muscle. For this reason, a change of surgical strategy allowed for preservation of the extensor digitorum communis muscle, which would otherwise have to be resected, with tendon transfer and successful restoration of the thumb abduction function.
\end{abstract}

Correspondence to: Professor Valerio D'Orazi, Department of General Microsurgery and Hand Surgery, Fabia Mater Hospital, Via Olevano Romano 25, I-00171 Rome, Italy

E-mail: info@valeriodorazi.com

Abbreviations: MFH, malignant fibrous histiocytoma; MRI, magnetic resonance imaging; EDC, extensor digitorum communis; CT, computed tomography; EMG, electromyography; NCCN, National Comprehensive Cancer Network; APL, abductor pollicis longus; PL, palmaris longus

Key words: malignant fibrous histiocytoma, sarcoma, forearm, microsurgical technique, tendon transfer

\section{Introduction}

Soft tissue sarcomas are rare tumors (1\% of all adult malignancies) with a dismal prognosis. Approximately $70 \%$ of sarcomas occur in the extremities $(49 \%$ in the lower and $19 \%$ in the upper limbs), while the remaining $30 \%$ occur in the trunk, abdomen or retroperitoneum $(1,2)$. Among the most common histological types of sarcomas of the extremities, malignant fibrous histiocytoma (MFH) is the one exhibiting the highest incidence, followed by liposarcoma, fibrosarcoma and neurofibrosarcoma, with a peak incidence in the sixth decade of life. According to the World Health Organization 2002 classification of soft tissue tumors (3), MFH may be categorized into three morphological subtypes: i) Storiform-pleomorphic MFH (undifferentiated high-grade pleomorphic sarcoma); ii) giant-cell MFH (undifferentiated pleomorphic sarcoma with giant cells); and iii) inflammatory MFH (undifferentiated pleomorphic sarcoma with prominent inflammation) (3). MFH is also considered by the American Joint Committee on Cancer to be a high-grade sarcoma by definition and, therefore, exhibiting high local aggressiveness.

The typical histological appearance of $\mathrm{MFH}$ is that of a hypercellular neoplasm, with marked nuclear and cytological pleomorphism, bizarre appearance of stromal cells and a fascicular or storiform model growth (4). The presence of a lesion suspicious for soft tissue sarcoma should prompt the surgeon to perform diagnostic-instrumental tests. X-ray may reveal ectopic calcifications in the surrounding tissues (5-20\%), sclerosis or clear erosion of adjacent bone due to tumor compression. Magnetic resonance imaging (MRI) may reveal a lobulated mass, with intermediate signal in both T1 and T2 images $(5,6)$. There are also frequent intracapsular areas of tumor necrosis and hemorrhage, which are occasionally so extensive that the tumor is mistakenly diagnosed as hematoma of the soft tissues (7).

A correct surgical approach is crucial in several tumors, as previously demonstrated by our studies $(8,9)$, and a survey of the literature suggests that surgery is the first choice for the treatment of MFH (10).

Owing to advances in surgical techniques, the advanced reconstructive methods and the development of effective adjuvant therapies, the use of widely demolitive surgery is 
now considered unnecessary. Over the last few decades, studies have demonstrated that conservative surgery, with or without adjuvant therapy, appears to be an effective treatment for sarcomas, including high-grade sarcomas, with a local recurrence rate of $7-15 \%$, with no significant differences in terms of overall survival and disease-free interval compared with amputation (11-13). Neoplastic infiltration of the resection margins at the end of surgery appears to be among the major factors affectng the rate of local recurrence. In fact, the local recurrence rate approximates $13 \%$ with margins of $<1 \mathrm{~cm}$, while it may be reduced to $0 \%$ in cases with margins of $>1 \mathrm{~cm}$.

In light of these data, amputation may no longer be considered to be the primary approach for the treatment of sarcomas of the limbs, but rather be reserved for those cases in which adequate resection cannot be achieved without severe aesthetic and functional deficits, or when the sarcoma is very proximally located in the limb. The functional compartmental resection represents the surgical evolution of amputation, although not without functional consequences. However, the literature over the last 20 years reveals that soft tissue sarcomas of the extremities and precisely localized within a muscle may be treated by excision of the entire affected muscle, with preservation of the surrounding innervated muscles, without performing total compartmental resection (14). The present study stems from a multidisciplinary management involving a team with expertise in general microsurgery, where microsurgical technique is routinely applied by the use of magnification devices such as Loupes 4.5x, operating microscope 4-40x, dedicated instruments and LED coaxial light.

\section{Case report}

A 79-year-old Caucasian man presented with a swelling in the middle third of the dorsal aspect of the left forearm. A core needle biopsy previously performed in a different hospital had not reached a definitive diagnosis. The patient also reported history of trauma 6 months earlier, with progressive volume increase, without pain or functional deficit. On clinical examination, the patient had a tense elastic $5-\mathrm{cm}$ swelling in the dorsal aspect of the left forearm (Fig. 1), hypomobile in the deep region, while the overlying skin appeared to be normal. The Tinel's sign and other tests assessing the functionality of the muscles and tendons of the hand and fingers were negative. The patient reported a dull pain elicited by palpation of the region in question and was subjected to instrumental diagnostic examinations.

Ultrasound imaging revealed a heterogeneous lesion with hyperechoic areas, perilesional hypervascularization and intralesional spots on signal-enhanced color Doppler sonography. An MRI revealed a neoformation sized $\sim 8 \times 4 \mathrm{~cm}$ within the extensor digitorum communis (EDC) muscle, without invasion of the interosseous space (Fig. 2). Bone involvement was also excluded by X-ray. Furthermore, the patient was subjected to total-body computed tomography (CT) staging that was negative for lymph node involvement or distant metastases. Finally, electromyography (EMG) was performed with normal findings, thus excluding any nerve compression or infiltration.

As the case history, physical examination and diagnostic tests were strongly suggestive of a tumor, we decided to perform

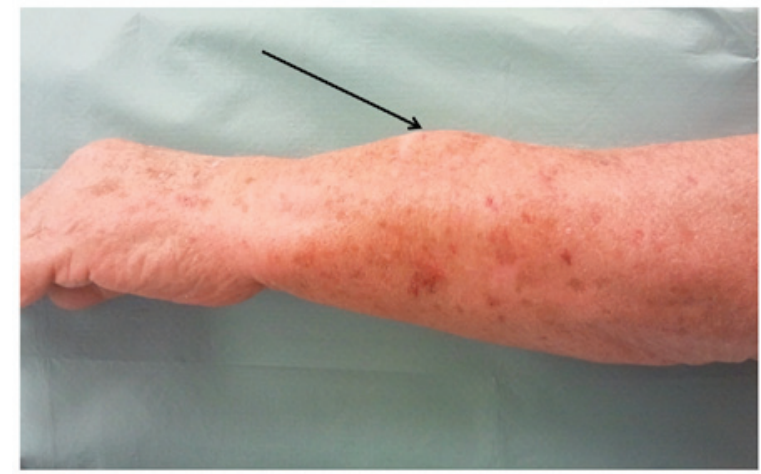

Figure 1. Preoperative macroscopic appearance, with a distinct elevated lesion in the dorsal aspect of the left forearm (arrow).

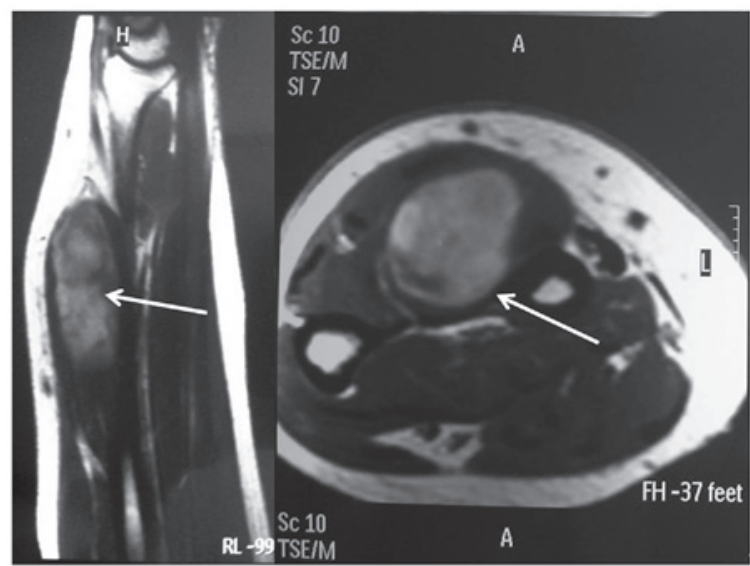

Figure 2. Magnetic resonance imaging (sagittal and axial planes) revealing a lesion in the left extensor digitorum communis muscle (arrows).

an open biopsy according to the criteria of Enneking et al (15). This procedure was performed under temporary ischemia of the arm, applying a tourniquet for $20 \mathrm{~min}$ at $220 \mathrm{mmHg}$ at the root of the arm. The bioptic specimen included skin, subcutaneous tissue, fascia, muscle and a portion of the tumor that appeared to be localized inside the muscle. Macroscopically, the neoformation was whitish and bled easily. Careful hemostasis was applied and the wound was closed without drainage. On histological examination, the neoformation exhibited irregular margins and consisted of spindle cells with nuclear atypia set in a dense, sclerotic, richly vascularized stroma, as evidenced by $\mathrm{CD} 34^{+}$staining. On immunohistochemical staining, the tumor was positive for CD68 and negative for smooth muscle actin (SMA) and S-100. The proliferative index, as assessed by $\mathrm{Ki}-67$, was $40 \%$ of the neoplastic elements. Thus, the final diagnosis was MFH. Based on the histological findings, MRI imaging and in accordance with the National Comprehensive Cancer Network (NCCN) guidelines (16), we decided to remove the tumor by excising the extensor compartment of the forearm with subsequent tendon transfer. Indeed, the guidelines suggest radical surgical treatment followed by radiotherapy for such soft tissue sarcomas.

A wide longitudinal incision was performed that also removed the scar of the earlier biopsy (Fig. 3). Surgery was performed by a microsurgical technique under magnification (Loupes 4.5x) and the use of dedicated instruments and 


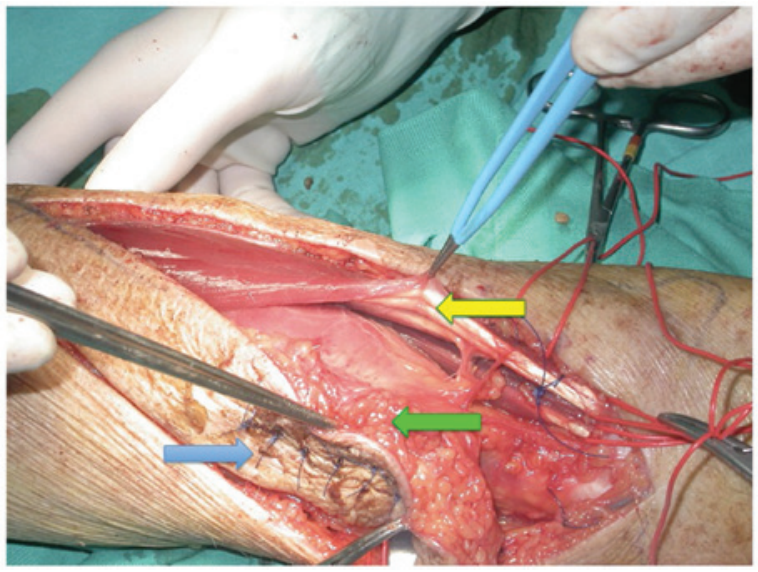

Figure 3. Surgical excision of the malignant fibrous histiocytoma. The blue arrow indicates previous biopsy scar, the green arrow indicates the tumor including in the APL muscle and the yellow arrow indicates EDC muscle preserved.

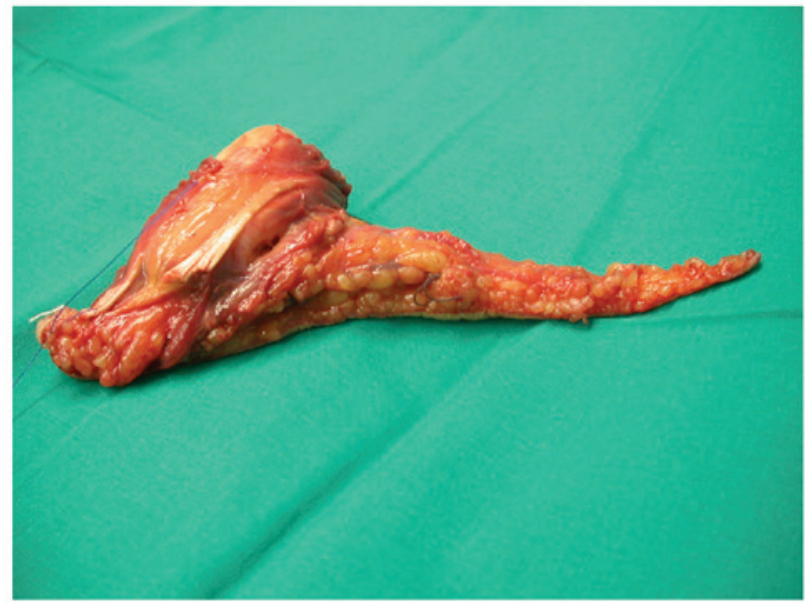

Figure 4. Malignant fibrous histiocytoma surgical specimen.

bipolar coagulator, allowing the identification of the correct anatomical cleavage planes and the preservation of the sensory branches of the radial nerve.

According to the MRI findings, the tumor was considered to be localized within the EDC muscle; however, the surgical exploration revealed that the lesion was located at a deeper plane, within the abductor pollicis longus muscle (APL). The muscle fibres appeared to be subverted and discontinued, while the APL muscle sheath did not exhibit macroscopic discontinuity or pathological adhesions to the adjacent and overlying muscles; the underlying bones did not appear to be infiltrated (Fig. 3). Therefore, we decided to change the surgical strategy and perform a wide excision of the tumor, including the APL muscle, without discontinuing the intact muscle sheath and the tumor pseudocapsule (Fig. 4), thus preserving the EDC muscle. Subsequently, through a palmar mini-incision, we performed a transfer of the palmaris longus (PL) tendon to the distal APL metacarpal tendon insertion with a Pulvertaft suture, in order to restore the abduction function of the thumb. The wound was sutured without a microsurgical flap coverage.

The histological examination revealed an encapsulated, whitish neoformation, sized $\sim 9 \times 4.5 \mathrm{~cm}$, with regular margins.

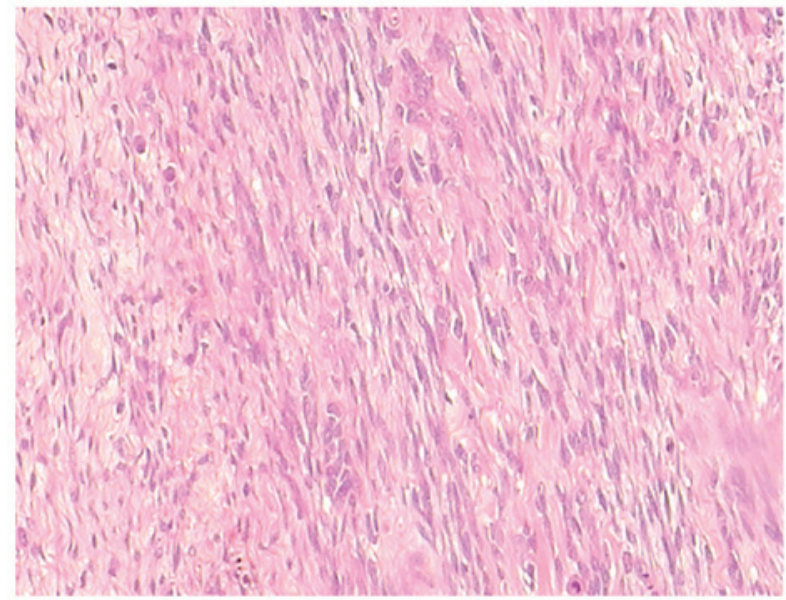

Figure 5. Malignant fibrous histiocytoma composed of neoplastic spindle cells with few extracellular connective matrix elements (hematoxylin and eosin staining; magnification, $\mathrm{x} 10$ ).

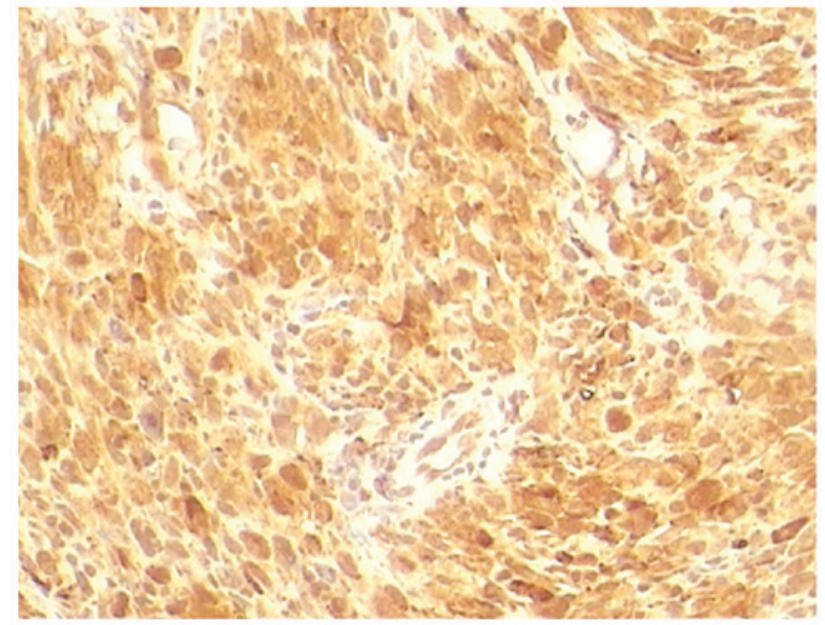

Figure 6. Neoplastic spindle cells exhibiting CD86 positivity (magnification, $\mathrm{x} 20)$.

Microscopically, the tumor consisted of spindle cells (Fig. 5) with nuclear atypia set in a dense, sclerotic, richly vascularised stroma, as evidenced by $\mathrm{CD}_{3} 4^{+}$staining. On immunoistochemical staining, the tumor was positive for CD68 (Fig. 6) and negative for SMA and S-100. The proliferative index, as assessed by $\mathrm{Ki}-67$, was $40 \%$ of the neoplastic elements. Therefore, a final diagnosis of MFH was made. The surgical resection margins and overlying skin were tumor-free. The MFH was classified as stage IIB (pT2b, pN0, pM0, G2 and R0) according to the American Joint Committee on Cancer (7).

After 2 weeks, the recovery of the strength and functional ability of the hand and thumb was satisfactory, compared to the contralateral side. After 2 months of rehabilitation, there was no functional deficit in the abduction of the thumb. In accordance with the NCCN guidelines (16), we consulted the oncologist and the radiologist, who did not suggest any adjuvant therapy, also taking into consideration the patient's comorbidities (prostate cancer, heart disease and chronic obstructive pulmonary disease). Moreover, the patient was informed of the options of adjuvant radiation therapy vs. observation and he 
selected observation. The patient has been followed up every 6 months for 4 years with clinical and instrumental examinations and he remains alive and recurrence-free.

\section{Discussion}

The diagnosis of soft tissue sarcomas located in the extremities is frequently delayed, as the tumor often grows slowly and is not associated with specific symptoms, apart from tension in the movements of the limb.

In the present case, the open biopsy according to the criteria of Enneking (15) established the diagnosis of MFH. The biopsy usually represents a strategy and not a small surgical intervention, therefore it is essential to first perform ultrasound, MRI or CT examinations to limit the risk of a biopsy procedure. A biopsy performed incorrectly may negatively affect subsequent therapy, increasing the risk of local recurrence and postoperative complications, worsening the functional outcome. According to the MRI findings, the tumor was considered to be localized within the EDC muscle. Therefore, surgery was planned to excise the tumor along with the EDC, which would mean complete detachment of digit tendons and severe functional damage. Accurate surgical dissection allowed us to identify the incorrect localization diagnosed by MRI, i.e., the sarcoma was unusually and unexpectedly located within the APL muscle. Therefore, we performed a wide excision of the sarcoma, including the APL muscle, without discontinuing the intact muscle sheath and the tumor pseudocapsule, preserving the EDC muscle. Simultaneous PL tendon transfer was performed, to restore the abduction function of the thumb. The surgical excision and reconstruction were performed by the same microsurgical team through the application of a microsurgical technique and the functional outcome was excellent. The follow-up at 4 years did not reveal tumor recurrence or metastatic spread.

In conclusion, when the sarcoma is entirely enclosed within a muscle, with free margins and without regional or distant metastases, a wide excision maintaining the muscle sheath intact may be considered as the optimal treatment.

\section{Acknowledgements}

The authors would like to thank the patient in this case report. Written informed consent was obtained from the patient for publication of his medical details and any accompanying images. A copy of the written consent is available for review by the Editor of this journal.

\section{References}

1. Weiss SW and Enzinger FM: Malignant fibrous histiocytoma: An analysis of 200 cases. Cancer 41: 2250-2266, 1978.

2. Gibbs JF, Huang PP, Lee RJ, McGrath B, Brooks J, McKinley B, Driscoll D and Kraybill WG: Malignant fibrous histiocytoma: An institutional review. Cancer Invest 19: 23-27, 2001.

3. Fletcher CDM, Unni KK and Mertens F (eds): Pathology and genetics of tumours of soft tissue and bone. In: World Health Organization Classification of Tumours. Pathology and Genetics of Tumours of Soft Tissue and Bone. IARC Press, Lyon, France, pp35-44, 2002.

4. Oda Y, Tamiya S, Oshiro Y, Hachitanda Y, Kinukawa N, Iwamoto Y and Tsuneyoshi M: Reassessment and clinicopathological prognostic factors of malignant fibrous histiocytoma of soft parts. Pathol Int 52: 595-606, 2002.

5. Nascimento AF and Raut CP: Diagnosis and management of pleomorphic sarcomas (so-called 'MFH') in adults. J Surg Oncol 97: 330-339, 2008.

6. Murphey MD, Gross TM and Rosenthal HG: From the archives of the AFIP. Musculoskeletal malignant fibrous histiocytoma: Radiologic-pathologic correlation. Radiographics 14: 807-826, quiz 827-828, 1994.

7. Goldblum JR, Folpe AL and Weiss SW: Rhabdomyosarcoma. In: Enzinger and Weiss's Soft Tissue Tumors. 6th edition. Saunders E (ed). Philadelphia, pp288-442, 2013.

8. Pasta V, Sottile D, Urciuoli P, Del Vecchio L, Custureri F and D'Orazi V: Rare chondrosarcoma of the breast treated with quadrantectomy instead of mastectomy: A case report. Oncol Lett 9: 1116-1120, 2015 .

9. Custureri F, D'Orazi V, Peparini N, Gabatel R, Urciuoli P, Patrizi G, Redler A and Di Matteo G: Choice of the surgical treatment in early gastric cancer. Hepatogastroenterology 51: 1210-1214, 2004.

10. Al-Refaie WB, Habermann EB, Jensen EH, Tuttle TM, Pisters PW and Virnig BA: Surgery alone is adequate treatment for early stage soft tissue sarcoma of the extremity. Br J Surg 97: 707-713, 2010.

11. Rosenberg SA, Tepper J, Glatstein E, Costa J, Baker A, Brennan M, DeMoss EV, Seipp C, Sindelar WF, Sugarbaker P, et al: The treatment of soft-tissue sarcomas of the extremities: Prospective randomized evaluations of (1) limb-sparing surgery plus radiation therapy compared with amputation and (2) the role of adjuvant chemotherapy. Ann Surg 196: 305-315, 1982.

12. Baldini EH, Goldberg J, Jenner C, Manola JB, Demetri GD, Fletcher CD and Singer S: Long-term outcomes after function-sparing surgery without radiotherapy for soft tissue sarcoma of the extremities and trunk. J Clin Oncol 17: 3252-3259, 1999.

13. Lin PP, Guzel VB, Pisters PW, Zagars GK, Weber KL, Feig BW, Pollock RE, Yasko AW: Surgical management of soft tissue sarcomas of the hand and foot. Cancer 95: 852-861,2002.

14. Pitcher ME and Thomas JM: Functional compartmental resection for soft tissue sarcomas. Eur J Surg Oncol 20: 441-445, 1994.

15. Enneking WF, Spanier SS and Goodman MA: A system for the surgical staging of musculoskeletal sarcoma. Clin Orthop Relat Res 153: 106-120, 1980.

16. National Comprehensive Cancer Network. NCCN Clinical Practice Guidelines in Oncology: Soft Tissue Sarcoma, Version 2.2008. http://www.calcuttayellow pages.com/ cimage25/109180sarcoma.pdf. Accessed July 17, 2015. 